\title{
PapMV nanoparticles improve mucosal immune responses to the trivalent inactivated flu vaccine
}

\author{
Gervais Rioux ${ }^{1 \dagger}$, Claudia Mathieu ${ }^{1 \dagger}$, Alexis Russell ${ }^{1}$, Marilène Bolduc ${ }^{1}$, Marie-Eve Laliberté-Gagné ${ }^{\prime}$ \\ Pierre Savard ${ }^{2}$ and Denis Leclerc ${ }^{1 *}$
}

\begin{abstract}
Background: Trivalent inactivated flu vaccines (TIV) are currently the best means to prevent influenza infections. However, the protection provided by TIV is partial (about 50\%) and it is needed to improve the efficacy of protection. Since the respiratory tract is the main site of influenza replications, a vaccine that triggers mucosal immunity in this region can potentially improve protection against this disease. Recently, PapMV nanoparticles used as an adjuvant in a formulation with TIV administered by the subcutaneous route have shown improving the immune response directed to the TIV and protection against an influenza challenge.
\end{abstract}

Findings: In the present study, we showed that intranasal instillation with a formulation containing TIV and PapMV nanoparticles significantly increase the amount of $\operatorname{lgG}$, IgG2a and IgA in lungs of vaccinated mice as compared to mice that received TIV only. Instillation with the adjuvanted formulation leads to a more robust protection against an influenza infection with a strain that is lethal to mice vaccinated with the TIV.

Conclusions: We demonstrate for the first time that PapMV nanoparticles are an effective and potent mucosal adjuvant for vaccination.

Keywords: PapMV nanoparticles, Adjuvant, Mucosal vaccine, Influenza, Trivalent inactivated flu vaccine, TIV, Seasonal flu vaccine, Mucosal immunity

\section{Background}

Vaccination with trivalent inactivated flu vaccines (TIV) remains the most affordable and efficient way to control diseases caused by influenza virus. The TIV is reformulated on the recommendation of $\mathrm{WHO}$ at the beginning of every year based on the circulating strains of the virus in the population. From that point, the manufacture process takes up to 8 months before the release the vaccine [1]. During this period, strains change through antigenic drift and shift and consequently, contribute to the decrease of vaccine effectiveness.

The TIV are currently administered by the intramuscular route, which is not optimal for protection to an influenza infection that occurs in the respiratory tract. The

\footnotetext{
*Correspondence: denis.leclerc@crchudequebec.ulaval.ca

${ }^{\dagger}$ Equal contributors

'Department of Microbiology, Infectiology and Immunology, 'Centre de recherche en Infectiologie', Laval University, 2705 boul. Laurier, Quebec City, PQ G1V 4G2, Canada

Full list of author information is available at the end of the article
}

favored route of vaccination to trigger mucosal immunity in the respiratory tract is the intranasal route $[2,3]$. However, the TIV was previously showed to be less immunogenic by the intranasal route $[4,5]$. The amount of antigen needed to trigger IgA production by this route must be increased by two fold which, directly impact the number of available doses $[4,5]$. Therefore, changes in the vaccine formulation to improve TIV efficacy in the respiratory tracts and increase the number of doses are needed [6].

Development of mucosal adjuvants that can improve both, the immune response to TIV in lungs and dose sparing became a priority $[5,7,8]$. Current available adjuvants are ineffective in inducing mucosal immunity because they cannot be administered by the intranasal route due to their biophysical properties. As an example, aluminium hydroxide, the most commonly used adjuvant in vaccines, is inefficient with TIV and cannot be used by the mucosal route [9]. New safe and potent adjuvants are therefore needed.

Ciomed Central

(c) 2014 Rioux et al.; licensee BioMed Central Ltd. This is an Open Access article distributed under the terms of the Creative Commons Attribution License (http://creativecommons.org/licenses/by/4.0), which permits unrestricted use, distribution, and reproduction in any medium, provided the original work is properly credited. The Creative Commons Public Domain Dedication waiver (http://creativecommons.org/publicdomain/zero/1.0/) applies to the data made available in this article, unless otherwise stated. 
In this study, we evaluated the potential of papaya mosaic virus (PapMV) nanoparticles to be used as a mucosal adjuvant. This hypothesis is supported by a previous report by our group showing that PapMV nanoparticles can trigger innate immunity in lungs of mice [10]. Instillation with PapMV nanoparticles triggered pro-inflammatory cytokines and chemokines secretion and immune cells recruitment shortly after treatment [10]. PapMV nanoparticles were also showed to be a TLR7 ligand, a receptor that activates innate immunity [11]. Furthermore, PapMV nanoparticles administered by the subcutaneous route were previously shown to improve and broaden the immune response to TIV in mouse and ferret animal models [7].

The objective of this study was to demonstrate that PapMV nanoparticles are able to induce mucosal immunity against TIV when administered by the intranasal route, in order to show its potential as a mucosal adjuvant. This route of administration was compared with subcutaneous injections as previously reported by our group [7].

\section{Methods}

\section{PapMV nanoparticles production}

PapMV nanoparticles were kindly provided by Folia Biotech Inc. (lot \# L-5728, Quebec City, Canada). Those PapMV nanoparticles are comparable to a lot used in previous studies [10]. In brief, PapMV nanoparticles are constituted of PapMV coat proteins and assemble in vitro around an RNA transcript. The Limulus Amebocyte lysate (LAL) assay (Lonza, Walkersville, Maryland, USA) was used to evaluate the levels of LPS contaminants that were found to be below the detection limit. PapMV nanoparticles were characterized by electron microscopy and dynamic light scattering as described previously [12]. In brief, nanoparticles sizes were recorded with a ZetaSizer Nano ZS (Malvern, Worcestershire, United Kingdom) at $0.1 \mathrm{mg} / \mathrm{ml}$ in $10 \mathrm{mM}$ Tris- $\mathrm{HCl}$ $\mathrm{pH}$ 8.0. Water diluted PapMV nanoparticles were stained with $3 \%$ acetate-uranyl on carbon-formvar grids then observed with a FEI-TECNAI-Spirit transmission electron microscope (FEI, Hillsboro, Oregon, USA).

\section{Vaccination protocol}

Mice, 6 to 8-week-old BALB/c (10/group), were immunized twice at 14-day interval by the intranasal (i.n.) or subcutaneous (s.c.) routes with $50 \mu$ l of a formulation containing $2.1 \mu \mathrm{g}$ of each hemagglutinin (HA) (TIV 2009-2010 (A/Brisbane/59/2007 (H1N1), A/Brisbane/ 10/2007 (H3N2), B/Brisbane/60/2008), cat \#9815, GlaxoSmithKline) alone or adjuvanted by $21 \mu \mathrm{g}$ of PapMV nanoparticles. Bleedings were performed at day 0 and 28 and bronchoalveolar lavages (BAL), at day 28 post-immunization.

\section{Antibody response quantification}

BALs were performed using $700 \mu \mathrm{l}$ of phosphate buffered saline and cells were purged from the samples by centrifugation. Blood sample were collected and centrifuged in BD Microtainer blood collection tubes (BD, Mississauga, Ontario, Canada) for 2 minutes at $10000 \times \mathrm{g}$. The harvested serums and BAL fluids were assayed for total IgG, IgG2a serotype and IgA against TIV or IgG2a against GST-NP by enzyme-linked immunosorbent assay (ELISA) as described elsewhere [7,13]. GST-NP is a C-terminal fusion of the influenza nucleoprotein to a glutathione S-transferase (GST) protein [7]. ELISAs were conducted by serial dilutions by two-fold steps starting at 1 in 50. Results are expressed as an antibody endpoint titer greater than threefold the background optical density values consisting of preimmune sera or control BAL fluids.

\section{Mice challenge with influenza virus}

Mice were challenged 2 weeks after the last immunization with 250 plaque-forming units (pfu) of A/WSN/ $33(\mathrm{H} 1 \mathrm{~N} 1)$ influenza virus by $50 \mu \mathrm{l}$ intranasal instillation. Weight losses and symptoms were monitored for 14 days post-infection. Symptoms are rated from 0 to 4 (no symptoms (0), lightly spiked fur and curved back [1], spiked fur and curved back [2], difficulty to move and light dehydration [3], severe dehydration, lack of reflex and ocular secretion [4]), where 4 is the highest score and mice are euthanized. Mice that lost more than 20\% of their initial weight are also euthanized.

\section{Statistical analysis}

Data from ELISA and challenge (day 8 weight losses and symptoms) were analyzed with a parametric ANOVA test. Tukey's post tests were used to compare differences among groups of mice. Kaplan-Meier survival curves were analysed by the log rank test. Values of $* \mathrm{p}<0.05$, $*$ p $<0.001$ and ${ }^{* * *} \mathrm{p}<0.0001$ were considered statistically significant. Statistical analyses were performed using GraphPad PRISM 5.01.

\section{Ethics statement}

All the work with animals has been done with institution approved ethics protocol by the "Comite de Protection des Animaux - CHUQ (CPA-CHUQ)". The approval of this project is found under the authorization number 2010-148.

\section{Results and discussion}

PapMV nanoparticles are composed of the recombinant coat proteins of PapMV purified from E. coli and selfassembled around a single-stranded RNA. Transmission electron microscopy revealed that PapMV nanoparticles have a filamentous rod shape structure (Figure 1A) and 
A

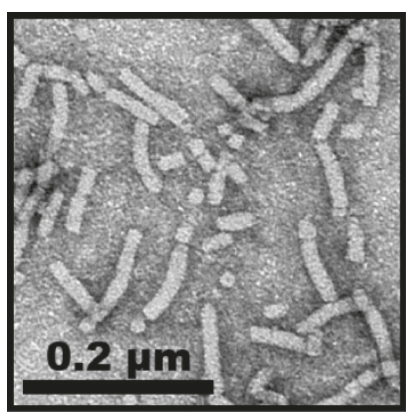

B

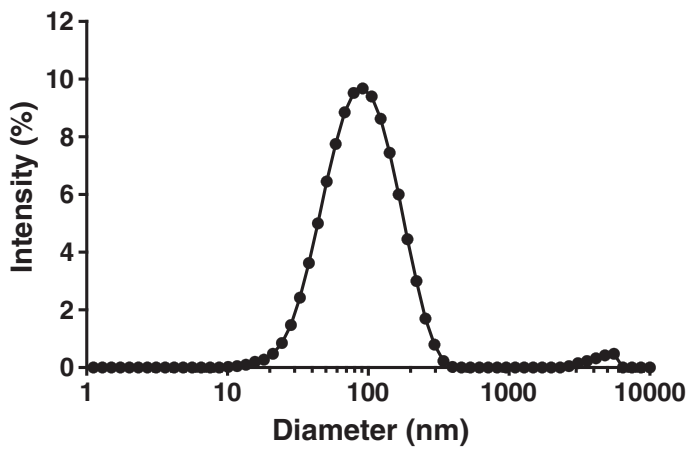

Figure 1 Structure of the PapMV nanoparticles. PapMV nanoparticles were observed with a transmission electron microscope and by dynamic light scattering. PapMV nanoparticles have a filamentous rod-shape structure (A) with an average length of $100 \mathrm{~nm}$ as shown by dynamic light scattering (B).
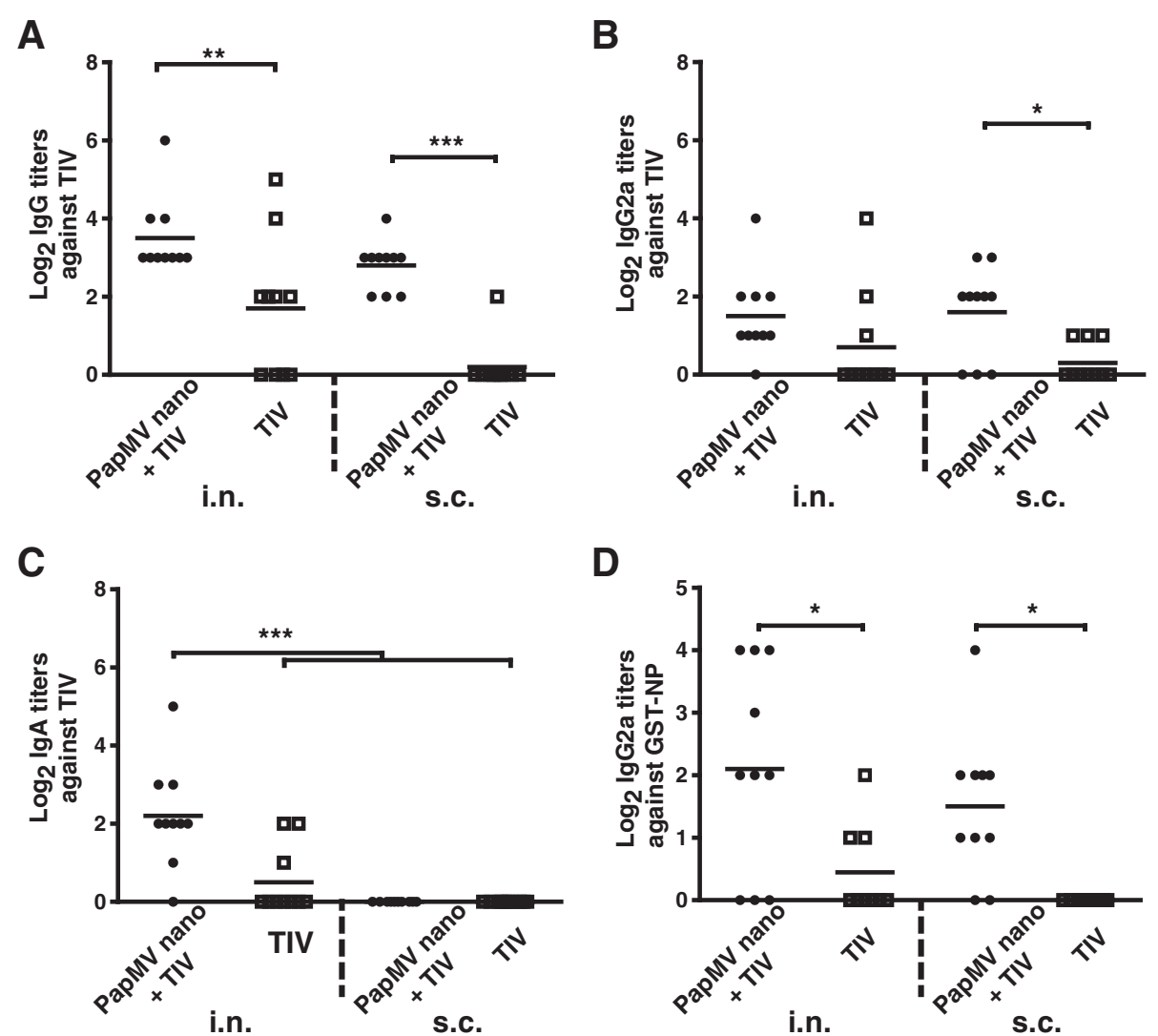

Figure 2 PapMV nanoparticles improve the mucosal antibody response against TIV and NP in the lungs. Balb/C mice (10/group) were vaccinated twice at a 14-day interval with TIV alone or adjuvanted by PapMV nanoparticles by intranasal (i.n.) or subcutaneous (s.c.) routes. Bronchoalveolar lavages were performed at day 28 and ELISA were conducted to evaluate the levels of total $\lg G$ (A), $\lg G 2 a$ (B) or $\lg A$ (C) against TIV and IgG2a against NP (D). Titers against TIV or GST-NP were not detected in the blood or BAL of PapMV nanoparticles or buffer vaccinated mice. ${ }^{*} \mathrm{P}<0.05,{ }^{* *} \mathrm{P}<0.01$ and ${ }^{* * *} \mathrm{P}<0.001$. 
an average length of $100 \mathrm{~nm}$, as confirmed by dynamic light scattering (Figure 1B).

To evaluate the potential of PapMV nanoparticles as a mucosal adjuvant, mice were immunized twice at 14 days interval by either the i.n. or s.c. routes with TIV alone or adjuvanted with PapMV nanoparticles. Antibodies levels against TIV in the BAL (Figure 2) and in the blood (Figure 3) were measured by ELISA at day 28. We showed that TIV adjuvanted with PapMV nanoparticles administered either by the i.n. or s.c. improved and potentiate the TIV as observed by the levels of total IgG titers directed against TIV (Figure 2A). IgG2a titers in the BAL showed significant higher levels in the aduvanted group only when administered by the s.c. route (Figure 2B). Interestingly, the group showing the highest IgA titers in the BAL was the adjuvanted TIV administered by the i.n. route (Figure 2C) showing clearly the effectiveness of PapMV nanoparticles to improve the mucosal antibody response in the lung. The ELISA also revealed that the adjuvanted vaccine broaden the immune response to the TIV antigens through a significant increased in the lungs of the IgG2a titers directed to the influenza NP (Figure 2D), a highly conserved protein through all the strains of influenza often used in experimental influenza vaccines $[7,14,15]$. NP has also been identified as a key antigen to trigger cross-protection to influenza viruses $[8,16-18]$. It is therefore a good strategy to increase the immune response directed to this antigen using PapMV nanoparticles to broaden and improve protection to this virus. Through the course of the experiment, we have not notice any sign of toxicity associated with the use of PapMV nanoparticles.

In the blood, the levels of total IgG or IgG2a were higher than in the BAL with either route of immunization (Figure 3). We also showed that the titers in total IgG or IgG2a directed to TIV or GST-NP were similar when the adjuvanted formulation was administered by the i.n. or the s.c. route (Figure 3A-B, D). As expected, only the adjuvanted vaccine administered by the i.n. route could trigger in the blood a significant amount of
A
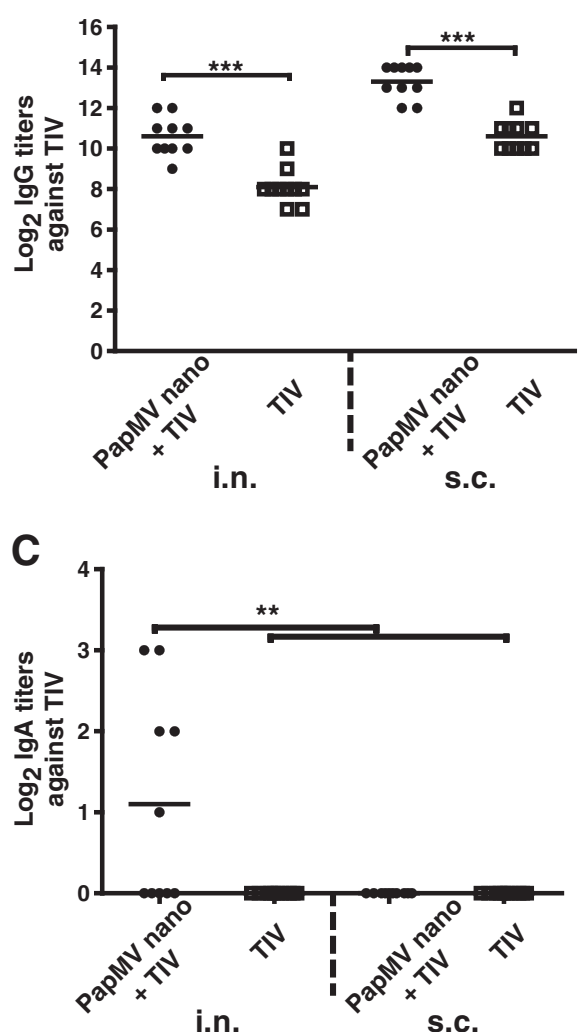

B
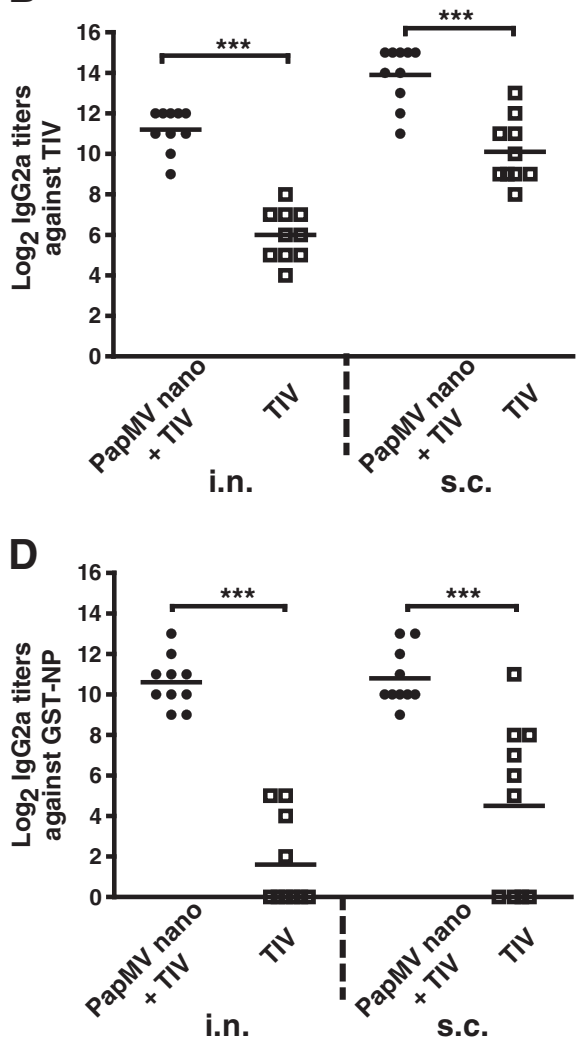

Figure 3 PapMV nanoparticles improve the systemic antibody response against TIV and NP. Balb/C mice (10/group) were vaccinated twice at a 14-day interval with TIV alone or adjuvanted with PapMV nanoparticles by intranasal (i.n.) or subcutaneous (s.c.) routes. Bleedings were performed at day 28 and ELISA were conducted to evaluate the levels of total IgG (A), IgG2a (B) or lgA (C) against TIV and IgG2a against NP (D) Titers against TIV or GST-NP were not detected in the blood or BAL of PapMV nanoparticles or buffer vaccinated mice. ${ }^{*} \mathrm{P}<0.05$, ${ }^{* *} \mathrm{P}<0.01$ and ***P $<0.001$. 


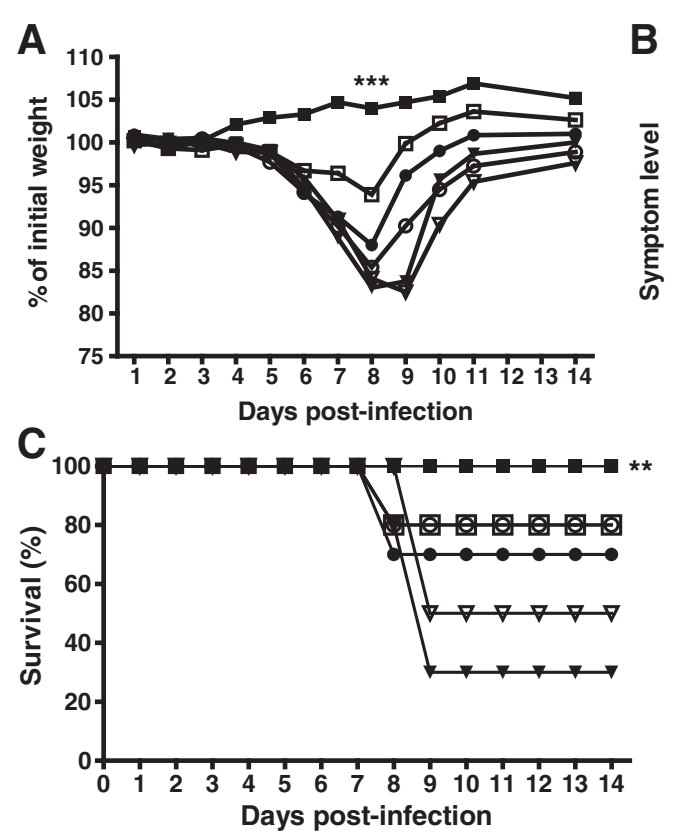

B

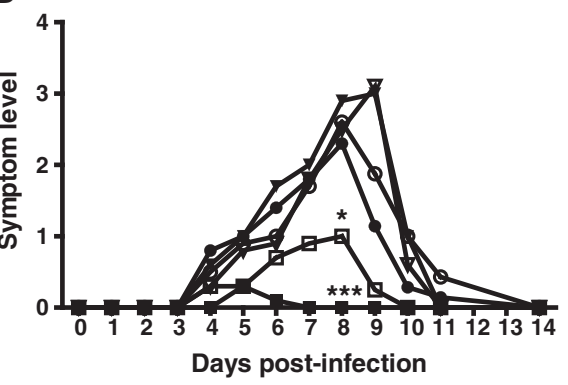

- PapMV nano + TIV i.n.

- TIV i.n.

$\rightarrow$ Buffer i.n.

曰 PapMV nano + TIV s.c.

$\theta$ TIV s.c.

$\nabla$ Buffer s.c

Figure 4 Mice vaccinated with PapMV nanoparticles adjuvanted TIV showed an increased protection to an influenza challenge with influenza strain normally lethal to TIV vaccinated mice. Mice vaccinated twice with TIV adjuvanted or not by PapMV nanoparticles by intranasal (i.n.) or subcutaneous (s.c.) routes were challenged with 250 pfu of ANSN/1933 (H1N1) influenza virus. Mice were monitored for weight loss (A), symptoms levels (B) and survival (C) for 14 days. Statistical analysis is applied between groups of the same immunization ways. ${ }^{*} \mathrm{P}<0.05$, ${ }^{* *} \mathrm{P}<0.01$ and ${ }^{* * *} \mathrm{P}<0.001$.

IgA directed to TIV (Figure 3C). Titers against TIV or GST-NP were not detected in the blood or BAL of PapMV nanoparticles or buffer vaccinated mice.

We previously showed that mice immunized s.c. with the TIV adjuvanted with PapMV nanoparticles induced protection against an strain of influenza that overcome the protection induced by the TIV [8]. In this study, we wish to validate that the adjuvanted TIV with PapMV nanoparticles administered by the intranasal route can also elicit this kind of protection. Therefore, we challenged vaccinated mice with $\mathrm{A} / \mathrm{WSN} / 33(\mathrm{H} 1 \mathrm{~N} 1)$, a strain of influenza that was previously showed to overcome the protection induced by the TIV. As expected, mice vaccinated with TIV by either route of immunization were not protected and showed major weight losses (more than 15\%) (Figure 4A) and strong symptoms (Figure 4B); while mice immunized by the i.n. route with the PapMV adjuvanted TIV showed a weight increased (Figure 4A) without any symptoms during infection (Figure 4B). Mice immunized with the same formulation by the s.c. route lost $7 \%$ of their initial weight and showed mild symptoms at the infection peak (day 8) (Figure 4A,B). Finally, only mice immunized by the i.n. route with the adjuvanted TIV formulation showed $100 \%$ survival (Figure 4C). The PapMV adjuvanted i.n. vaccine generated a robust protection to the infection by an influenza strain normally lethal to TIV vaccinated mice.
PapMV nanoparticles is therefore an efficient mucosal adjuvant with strong potential for its used in human.

\section{Conclusions}

In conclusion, we demonstrate for the first time that PapMV nanoparticle is an effective and potent mucosal adjuvant for vaccination against a respiratory disease. This technology fulfills an important medical need for a safe mucosal adjuvant that broadens the protection of the TIV.

\section{Abbreviations \\ PapMV: Papaya mosaic virus; TIV: Trivalent inactivated flu vaccines; \\ HA: Hemagglutinin; BAL: Bronchoalveolar lavage; ELISA: Enzyme-linked immunosorbent assay; NP: Nucleoprotein; GST: Glutathione S-transferase; Pfu: Plaque-forming units; i.n: Intranasal; s.c: Subcutaneous.}

\section{Competing interests}

Author Denis Leclerc and Pierre Savard are shareholder of the company FOLIA BIOTECH INC., a start-up company that has the mandate to exploit commercially this technology to improve and design new vaccines. This does not alter the authors' adherence to all the journal policies.

\section{Authors' contributions}

GR performed mice experiments and drafted the manuscript. CM performed mice experiments and immunoassays and helped to draft the

manuscript. GR and CM are considered to have equally contributed to this article. AR performed mice experiments and helped to draft the manuscript. MB, MELG and PS engineered the nanoparticles. DL supervised the study and revised the manuscript. All authors read and approved the final manuscript. 


\section{Acknowledgment}

We would like to thank the "Plateforme de bio-imagerie du centre de recherche en infectiologie" for letting us use the transmission electron microscope. This work was supported by a grant from the Canadian Institute of Health Research [185160] and FOLIA BIOTECH INC.

\section{Author details}

'Department of Microbiology, Infectiology and Immunology, 'Centre de recherche en Infectiologie', Laval University, 2705 boul. Laurier, Quebec City, PQ GIV 4G2, Canada. ${ }^{2}$ Neurosciences, Laval University, Quebec City, PQ, Canada.

Received: 4 March 2014 Accepted: 23 April 2014

Published: 3 May 2014

\section{References}

1. Barr IG, McCauley J, Cox N, Daniels R, Engelhardt OG, Fukuda K, Grohmann G, Hay A, Kelso A, Klimov A, Odagiri T, Smith D, Russell C, Tashiro M, Webby R, Wood J, Ye Z, Zhang W: Epidemiological, antigenic and genetic characteristics of seasonal influenza $A(\mathrm{H} 1 \mathrm{~N} 1), A(\mathrm{H} 3 \mathrm{~N} 2)$ and $B$ influenza viruses: basis for the WHO recommendation on the composition of influenza vaccines for use in the 2009-2010 Northern Hemisphere season. Vaccine 2010, 28(5):1156-1167.

2. Keitel WA, Cate TR, Nino D, Huggins LL, Six HR, Quarles JM, Couch RB: Immunization against influenza: comparison of various topical and parenteral regimens containing inactivated and/or live attenuated vaccines in healthy adults. J Infect Dis 2001, 183(2):329-332.

3. Belyakov IM, Ahlers JD: What role does the route of immunization play in the generation of protective immunity against mucosal pathogens? $J$ Immunol 2009, 183(11):6883-6892.

4. Atmar RL, Keitel WA, Cate TR, Munoz FM, Ruben F, Couch RB: A doseresponse evaluation of inactivated influenza vaccine given intranasally and intramuscularly to healthy young adults. Vaccine 2007, 25(29):5367-5373.

5. Hong SH, Byun Y-H, Nguyen CT, Kim SY, Seong BL, Park S, Woo GJ, Yoon Y, Koh JT, Fujihashi K, Rhee JH, Lee SE: Intranasal administration of a flagellin-adjuvanted inactivated influenza vaccine enhances mucosal immune responses to protect mice against lethal infection. Vaccine 2012, 30(2):466-474.

6. Woodrow KA, Bennett KM, Lo DD: Mucosal vaccine design and delivery. Annu Rev Biomed Eng 2012, 14:17-46.

7. Savard C, Guérin A, Drouin K, Bolduc M, Laliberté-Gagné M-E, Dumas M-C, Majeau N, Leclerc D: Improvement of the trivalent inactivated flu vaccine using PapMV nanoparticles. PLoS One 2011, 6(6):e21522.

8. Langley JM, Aoki F, Ward BJ, McGeer A, Angel JB, Stiver G, Gorfinkel I, Shu D, White L, Lasko B, Dzongowski P, Papp K, Alexander M, Boivin G, Fries L: A nasally administered trivalent inactivated influenza vaccine is well tolerated, stimulates both mucosal and systemic immunity, and potentially protects against influenza illness. Vaccine 2011, 29(10):1921-1928.

9. Lawson LB, Norton EB, Clements JD: Defending the mucosa: adjuvant and carrier formulations for mucosal immunity. Curr Opin Immunol Elsevier Ltd 2011, 23(3):414-420.

10. Mathieu C, Rioux G, Dumas M-C, Leclerc D: Induction of innate immunity in lungs with virus-like nanoparticles leads to protection against influenza and Streptococcus pneumoniae challenge. Nanomedicine 2013, 9:839-848.

11. Lebel M-Ë, Daudelin J-F, Chartrand K, Tarrab E, Kalinke U, Savard P, Labrecque N, Leclerc D, Lamarre A: Nanoparticle Adjuvant Sensing by TLR7 Enhances CD8+ T Cell-Mediated Protection from Listeria Monocytogenes Infection. J Immunol 2014, 192(3):1071-1078.

12. Rioux G, Majeau N, Leclerc D: Mapping the surface-exposed regions of papaya mosaic virus nanoparticles. FEBS J 2012, 279(11):2004-2011.

13. Rioux G, Babin C, Majeau N, Leclerc D: Engineering of papaya mosaic virus (PapMV) nanoparticles through fusion of the HA11 peptide to several putative surface-exposed sites. PLoS One 2012, 7(2):e31925.

14. Vemula SV, Ahi YS, Swaim A-M, Katz JM, Donis R, Sambhara S, Mital SK Broadly protective adenovirus-based multivalent vaccines against highly pathogenic avian influenza viruses for pandemic preparedness. PLoS One 2013, 8(4):e62496.

15. Wang W, Huang B, Jiang T, Wang X, Qi X, Gao Y, Yan W, Ruan L: Robust immunity and heterologous protection against influenza in mice elicited by a novel recombinant NP-M2e fusion protein expressed in E. coli. PLoS One 2012, 7(12):e52488.

16. Carragher DM, Kaminski DA, Moquin A, Hartson L, Randall TD: A novel role for non-neutralizing antibodies against nucleoprotein in facilitating resistance to influenza virus. J Immunol 2008, 181(6):4168-4176.

17. LaMere MW, Lam H-T, Moquin A, Haynes L, Lund FE, Randall TD, Kamiski DA: Contributions of antinucleoprotein IgG to heterosubtypic immunity against influenza virus. J Immunol 2011, 186(7):4331-4339.

18. Lamere MW, Moquin A, Lee FE-H, Misra RS, Blair PJ, Haynes L, Randall TD, Lund FE, Kaminski DA: Regulation of antinucleoprotein IgG by systemic vaccination and its effect on influenza virus clearance. J Virol 2011, 85(10):5027-5035.

doi:10.1186/1477-3155-12-19

Cite this article as: Rioux et al:: PapMV nanoparticles improve mucosal immune responses to the trivalent inactivated flu vaccine. Journal of Nanobiotechnology 2014 12:19.

\section{Submit your next manuscript to BioMed Central and take full advantage of:}

- Convenient online submission

- Thorough peer review

- No space constraints or color figure charges

- Immediate publication on acceptance

- Inclusion in PubMed, CAS, Scopus and Google Scholar

- Research which is freely available for redistribution 